\title{
205 Sphincteric functions of the larynx
}

A The aryepiglottic sphincter closes during deglutition and vomiting.

B The false cord sphincter is primarily involved in preventing ingress of foreign material.

C The true vocal cords have a curved inferior surface with the concavity directed inferiorly.

D The false cord sphincter cannot be closed independently of the true vocal cords.

E The mechanical mechanism of the true vocal cord sphincter can resist a pharyngeal pressure of $150 \mathrm{~mm} \mathrm{Hg}$.

\section{During deglutition}

A Only the aryepiglottic sphincter closes.

B The larynx is lowered to assist passage of the food bolus into the pharyngo-oesophageal opening.

C Laryngeal airflow continues in an outward direction.

D The epiglottis tilts forwards.

E The lymphocytes in the tonsils sample the food as it passes by. 